\title{
Cumulant Analysis of Rössler Attractor and its Applications
}

\author{
V. Kontorovich ${ }^{*, 1}$, L. A. Beltrán ${ }^{2}$, Jorge Aguilar ${ }^{2}$, Z. Lovtchikova $^{3}$ and Keith R. Tinsley ${ }^{4}$ \\ ${ }^{I}$ Electrical Engineering Department, Telecommunications Section, CINVESTAV-IPN Av. IPN, Col. San Pedro \\ Zacatenco, C.P. 07360, D.F. México \\ ${ }^{2}$ Intel Tecnologías de México, Parque Industrial. Tecnológico II Periférico Sur, 7980, Jalisco, México \\ ${ }^{3}$ Interdisciplinary Professional Unit in Engineering and Advanced Technologies, UPIITA-IPN Av. IPN, Col. Ticoman, \\ C.P. 07340, D.F. México \\ ${ }^{4}$ Intel Labs, Intel Corporation Hillsboro, Oregon 97124, USA
}

\begin{abstract}
This paper is dedicated to the statistical analysis of the chaos, generated by Rössler attractor, based on the socalled "Degenerated Cumulant Equations" method. The approach is illustrated by the calculus of the first cumulants, which are necessary to create an approximation of the Probability Density Function (PDF), applying the Gramm-Charlier series, the model distribution method, etc. An approximate method for the variance calculation at the output of the Rössler strange attractor is presented as well. The latter is based on the Kolmogorov-Sinai entropy estimated by the Lyapunov exponents for a statistically linearized chaotic system. Finally, an application using Rössler attractor output signals to model Radio Frequency Interferences (RFI) provided by Peripherical Component Interface express (PCIe) serial bus of high speed interconnects of Laptops and Desktops is presented.
\end{abstract}

Keywords: Cumulants, Cumulant brackets, Attractor, Kurtosis, Variance, Kolmogorov-Sinai entropy.

\section{INTRODUCTION}

In recent times the applications of chaos theory in the electrical engineering field has grown considerably [1-5] and some fresh and very interesting reviews, related to the statistical analysis of strange attractors (continuous nonlinear dynamic systems generating chaos) (see [6, 7], and references therein) have been published. However, those comprehensive reviews of chaos have been written "by physicists for physicists" and do not contain the material necessary for the engineering statistical analysis of the chaotic attractors. For application purposes it is necessary to have a simple and rather accurate methodology to evaluate the statistical characteristics of the attractors due to their circuit parameters, adjusting such parameters to the required features of the chaotic models, etc. It is worth to stress here, that engineering methods for the statistical analysis of attractors are still missing.

In an effort to fill this gap and provide an adequate tool for the applied statistical analysis of strange attractors, the so-called "degenerated cumulant equations method" was recently proposed, based entirely on the parameters of the corresponding dynamic systems ${ }^{1}$. As it was shown in those studies, not only the expressions for the cumulants can be

*Address correspondence to this author at the Electrical Engineering Department, Telecommunications Section, CINVESTAV-IPN Av. IPN, Col. San Pedro Zacatenco, C.P. 07360, D.F. México;

E-mails: valeri@cinvestav.mx, lcruzb06@ipn.mx

\footnotetext{
${ }^{1}$ Certainly, all statistical properties of the attractor can be obtained through
} statistical computer simulation, but it is not the case here. found by means of the proposed approach, but also the socalled "model distributions" for each component of the attractors under analysis can be constructed.

Compared against the moments, the cumulants have very attractive features, which can be exploited for engineering applications, as it is carefully explained in $[8,9]$; also in these works a comprehensive and adequate method for the cumulant calculus was presented: the cumulant brackets.

Since the first time the cumulants were used in applications it was noticed that they are actually a set of "independent statistical coordinates" of the random process and thus provide a "general view" of the statistical behavior of the system due to the system parameters $[9,10]$, etc.

It is interesting to notice that in practical applications the "weight" or influence of the cumulants becomes negligible as the order of the cumulants grows [9]. For engineering analysis this translates to the fact that it is often enough to consider only the first four cumulants: $\chi_{1}-\chi_{4}$, and the corresponding shape coefficients for the PDF namely, $\gamma_{3}$ (the skewness coefficient) and $\gamma_{4}$ (the kurtosis coefficient) [9]. Notice that the cumulant analysis of the attractors is both qualitative and quantitative, and the "balance" between these two features highly depends on the type of attractor and its parameters. This will be illustrated taking the Rössler attractor as an example.

Regarding applications, it was recently proved that chaotic systems are adequate to model several natural phenomena related to the communication field: for example, the output signals of some components of well known attractors (Lorenz, Chua, etc.) can adequately describe the 
PDFs of the interferences generated by some digital interconnects, and the cumulant analysis provides a good coincidence with the measurement data [11].

Certainly, chaotic signals can not be applied for "physical "modeling of the radio interference phenomena, but are rather adequate for a statistical modeling approach.

The improvement on performance of modern digital communications, particularly from mobile to mobile PC users, has turned so important that, it results highly encouraging to continue with the search of other attractors, for example, the Rössler attractor, as perspective models to characterize the interferences from the digital interconnects as well.

As it follows from the title, this paper is dedicated to the cumulant analysis of the Rössler attractor and its application to get a statistical modeling of the RFI produced by digital interconnectors of laptops and desktops. The paper is organized as follows. Section II contains some basics of the chaos and its description, but it is mainly dedicated to the degenerated cumulant method. Section III is dedicated to theoretical analysis (based on the degenerated cumulant equations) of the Rössler attractor. In section IV some of the numerical results for Rössler attractor and their comparison with analytical predictions are presented. In section $\mathrm{V}$ an approximate method to evaluate the variance for the Rössler attractor and its generalization is shown. Section VI presents the material of the applications of the output signals of Rössler attractor for the statistical (RFI) modeling from PCIe. Finally, some conclusions are mentioned in section VII.

\section{BASICS OF THE CHAOTIC ATTRACTORS THEORY. DEGENE- RATED CUMULANT EQUATIONS APPROACH.}

It is well known [12], that each dissipative continuous time dynamic system (strange attractor) can be defined with the following equation:

$\dot{\mathbf{x}}=\mathbf{f}(\mathbf{x}(t)), \quad \mathbf{x} \in \mathbf{R}^{n}, \quad \mathbf{x}\left(t_{0}\right)=\mathbf{x}_{0}$,

where $\mathbf{f}(\cdot)=\left[f_{1}(\mathbf{x}), \ldots, f_{\mathrm{n}}(\mathbf{x})\right]^{\mathrm{T}}$ is a differentiable vector function.

Chaotic attractors, described by (1) can be classified as hyperbolic, quasi-hyperbolic and non-hyperbolic [6, 7]. All attractors, which were statistically investigated beforehand (see, for example $[1,6-8,11,13]$ ) are of the quasi-hyperbolic or non-hyperbolic type: (Lorenz, Chua, Rössler, etc.). The quasi-hyperbolic attractors (Lorenz attractor with the typically considered parameters $[1,6-8,11,13])$ do not actually differ from the hyperbolic (robust, ideal) attractors and existence of the invariant measure for them is practically guaranteed (see detail at $[6,7]$ ).

In the following, we shall explicitly follow the principles of ergodic theory for equations such as (1) [12], assuming the existence of an invariant measure for solutions of (1). As an invariant measure we will take a physical measure (see sections II-F and IV-H in [12]), applying the following idea of Kolmogorov: in (1) one has to consider a weak external noise $\xi(\mathrm{t})$, i.e. $\dot{\mathbf{x}}=\mathbf{f}(\mathbf{x}(t)+\varepsilon \xi(t))$

where $\xi(t)$ is a vector of a weak external white noise with the related positive defined matrix of "intensities" $\varepsilon=\left[\varepsilon_{\mathrm{ij}}\right]^{\mathrm{nxn}},[7$, $8]$.

Here it is reasonable to give some comments regarding the concepts of the invariant and physical measures. Invariant measure is a measure for the "space" averaging of the chaos realizations: it is independent to initial distributions, it completely specifies the statistical characteristics of the attractor, and it is not unique $[6,7,12]$. Physical measure, which appears from the time averaging for each ergodic chaos realization is obviously more reasonable from the engineering point of view and as it was mentioned before actually it follows from the Kolmogorov's idea (2).

Now it is clear, that in the framework of the ergodic theory invariant and physical probabilistic measures might be synonyms, but physical measure is more relevant for the applications.

Next, it can be seen that (2) is a stochastic differential equation (SDE), generating the continuous Markov process with a physical measure (PDF) $W \varepsilon(\mathbf{x}, \mathrm{t})$ [9].

The relationships between chaotic continuous processes and Markov processes were completely discussed in $[6,7$, $14,15]$, etc; we will only stress here, that the linear operator for the transient PDF $W_{\varepsilon}(\mathbf{x}, \mathrm{t})$ (so-called Fokker-PlanckKolmogorov) for SDE (2) has the same properties as the FPQ operator [14, 15] for discrete chaos and provides a stationary distribution: $\lim _{\mathrm{t} \rightarrow \infty} \mathrm{W}_{\varepsilon}(\mathbf{x}, \mathbf{t})=\mathrm{W}_{\mathrm{st}}^{\varepsilon}(\mathbf{x}, \mathbf{t})$ when the vector function $\mathbf{f}(\cdot)$ does not depend on time ' $t$ ' (sufficient conditions, see [9]).

Thus, the physical measure $W_{\text {st }}(\mathbf{x})$ has to be taken as:

$\mathrm{W}_{\mathrm{st}}(\mathbf{x})=\lim _{\forall \varepsilon_{\mathrm{ij}} \rightarrow 0} \mathrm{~W}_{\mathrm{st}}^{\varepsilon}(\mathbf{x})$.

Assumption (3) is important for all subsequent analysis, as we expect that when all elements of matrix " $\boldsymbol{\varepsilon}$ " equally tend to zero, the solution of (1) and (2) has the same $W_{s t}(\mathbf{x})$. In other words $W_{s t}(\mathbf{x})$ is defined in asymptotic conditions of weak noise in (2). This noise is not certainly a 'white' one (Kolmogorov mentioned 'some noise'), thus it might be Poisson noise as well, but hereafter we will assume that it is a white noise in (2).

Now, let us make some particular comments to this assumption for (2), if (2) describes the chaotic attractor. The Markov process, when noise intensities in SDE (2) tend to zero is a so-called "quasi-deterministic" or "degenerated" Markov process and it is pretty hard to obtain the closed form expression for a physical measure in (3), if " $n$ " in (1) is more than one. This issue was somehow briefly touched at [16].

Does it mean then, that the $W_{s t}(\mathbf{x})$ do not exist? Of course not, it does exist, but the Markov description of the chaos simply might not provide us with the adequate closed form representation of the physical measure $W_{s t}(\mathbf{x})$. In contrary, as it was shown in [8] and will be presented in the following, 
the "degenerated cumulant equations" approach provides us closed form solutions for the "degenerated" case.

The non-hyperbolic attractors are rather sensitive to variations of their parameters: the character of chaos can change drastically (from spiral to punnel [7, 17]), for example, for Rössler attractor when the " $c$ " parameter (see below) changes from 5.7 to 10 . Nevertheless the existence of the stationary measure for the Rössler attractor was confirmed in [7]. The same matter was checked experimentally by the authors for the Chua attractor case.

Please note that to the best of our knowledge, the detailed study of the existence of the stationary physical measure for the non-hyperbolic attractors is rather far from over. Next, it is worth mentioning here that the problem of the relaxation of output chaotic signals of the attractors to it is stationary conditions still remains open $[6,7]$.

We propose in the following (section $\mathrm{V}$ ) as a first approximation for the time constant to use the inverse of the eigenvalues of the statistically linearized matrix of the attractor. Sure, this approximation is only valid for the experimental evaluation of the first cumulants.

The $W_{s t}(\boldsymbol{x})$, as well as its characteristic function, is totally defined by the complete set of cumulants [9] and we will show in the sequel, that conditions as in (3) are valid for all stationary cumulants of the solutions of (1) and (2).

Then: $W_{s t}(\mathbf{x})=F^{-1}\{\theta(j v)\}$, where $\theta(j v)$ is a characteristic function and $F\{\cdot\}$ and $F^{-1}\{\cdot\}$ are direct and inverse Fourier transforms respectively:

$\theta(j v)=\exp \left\{\sum_{\mathrm{s}=1}^{\infty} \frac{\mathrm{j}^{\mathrm{s}}}{\mathrm{s} !} \chi_{\mathrm{m}_{1}, \mathrm{~m}_{2}, \ldots, \mathrm{m}_{\mathrm{n}}}^{\xi_{1}, \xi_{2}, \ldots, \xi_{\mathrm{n}}} v_{1}^{\mathrm{m}_{1}} \ldots v_{\mathrm{n}}^{\mathrm{m}_{\mathrm{n}}}\right\}$

where $\left\{\xi_{\mathrm{i}}\right\}_{1}^{n}$ are random variables, $m_{1}+m_{2}+\ldots+m_{n}=S$, $\chi_{m_{1}, \ldots, m_{n}}^{\xi_{1}, \ldots, \xi_{n}}$ is the joint cumulant of the $s$-th order;

$v=\left[v_{1, \ldots,}, v_{\mathrm{n}}\right]^{\mathrm{T}}$.

As it was stated above and follows from (4), (see also [8, 10]) the full set of cumulants $\chi_{m_{1}, \ldots, m_{n}}^{\xi_{1}, \ldots, \xi_{n}}$ completely represent $W_{s t}(\mathbf{x})$, as long as the series (4) converges for all $\left\{v_{i}\right\}_{i=1}^{n}$.

Therefore, the analysis of cumulants renders the same results as the analysis of $W_{s t}(\mathbf{x})$ and the advantages of cumulant versus moments for this goal are well known [10, $18]$.

It is worth mentioning here that the formalism for cumulant calculus for random variables and stochastic processes was proposed by A. N. Malakhov ([10] see also [9]). The idea of applying the cumulant analysis for chaos dynamical systems was presented in $([8,16]$ see also the references therein). In the following, for calculations of $W_{s t}(\mathbf{x})$ we will evoke some ideas of Malakhov [10]: model distributions, cumulant analysis and cumulant equations.
If one assumes that cumulants for all $s>m$ are equal to zero, then for the finite set of cumulants $\left\{\chi_{s}\right\}_{1}^{m}$, we can introduce the "model distribution" $\hat{W}_{s t}(\mathbf{x})$ of the $m$-th order and its characteristic function defined by $\theta_{m}(j \mathrm{v})$.

For example, for the characteristic function of one dimensional model distribution (or model approximation) the following representation is valid:

$$
\begin{aligned}
& \hat{W}_{s t}(x)=F^{-1}\left\{\theta_{m}(j v)\right\} \\
& \theta_{m}(j v)=\exp \left\{\sum_{s=1}^{m} \frac{(j v)^{s}}{s !} \chi_{s}\right\},
\end{aligned}
$$

where $\chi_{s}$ is the cumulant of the $s$-th order.

Note, that the distribution $\hat{W}_{s t}(\mathrm{x})$ is only an approximation of the true PDF and the model distributions $([9,10]$, and references therein) provide an accuracy, better than the orthogonal series expansions [9] for the case $\gamma_{4}<0$, but it can also be applied for $\gamma_{4}>0$.

Another option for analytical approximation for $W_{s t}(\mathrm{x})$ is the orthogonal series representations, for example, GrammCharlier, Laguerre series, etc. [9].

For example, the Gramm-Charlier series are defined by:

$$
W(x)=W_{G}(x)\left[1+\frac{\gamma_{3}}{3 !} H_{3}(x)+\frac{\gamma_{4}}{4 !} H_{4}(x)\right],
$$

where

$$
H_{n}(x)=(-1)^{n} \exp \left\{\frac{x^{2}}{2}\right\} \frac{d^{n}}{d x^{n}} \exp \left\{-\frac{x^{2}}{2}\right\},
$$

$H_{n}(\cdot)$ is a Hermitian polynomial of $n$-order,

$W_{G}(x)=\frac{1}{\sqrt{2 \pi}} \exp \left\{-\frac{x^{2}}{2}\right\}$,

is a Gaussian distribution,

$$
\gamma_{3}=\frac{\chi_{3}}{\sigma^{3}}
$$

is the skewness coefficient and

$$
\gamma_{4}=\frac{\chi_{4}}{\sigma^{4}}
$$

is the kurtosis coefficient.

It is important to mention that for a symmetrical PDF the above coefficients satisfy:

$$
\gamma_{3}=0, \mu_{3}=\chi_{3}, \mu_{4}=\chi_{4}+3\left(\sigma^{2}\right)^{2},
$$

with $\chi_{3}$ and $\chi_{4}$ being the third and fourth cumulants; $\gamma_{4} \geq-2$.

As it renders from the material presented above, (1) can be rewritten in the form of the stochastic differential equation (SDE); in other words $\mathbf{x}(\mathrm{t})$ is a continuous $n$ - 
dimensional Markov process with kinetic coefficients, given by $\mathbf{K}_{1_{i}}(\mathbf{x})=\mathbf{f}_{\mathbf{i}}(\mathbf{x})$ and $\mathbf{K}_{2}=\left[\varepsilon_{i j}\right]^{n x n},[8,9]$.

For the SDE representation of the attractor the approach, named as cumulant equations for the SDE with the given $\mathbf{K}_{1_{i}}(\mathbf{x})$ and $\mathbf{K}_{2}[8,9]$, can be successfully applied.

Next, let us remind the assumption (as it was proposed above), that $W_{s t}(\mathbf{x})$ exists, and that it is a reasonable physical measure for (1) and (2), although there exists all cumulants that adequately represent $W_{s t}(\mathbf{x})$.

Let us present here briefly the procedure to develop the cumulant equations from SDE (2) (the interested reader can find details at $[9,10])$. It is well known, that $W(\boldsymbol{x}, t)$ for the solution of (2) follows the so-called Fokker-PlankKolmogorov Equation (FPK, or direct Kolmogorov Equation), whose operator is self ad-joint to the operator of the so-called inverse Kolmogorov Equation (see [9, 10], for definitions and details). This inverse Kolmogorov Equation has the following operator for $\operatorname{SDE}(2)[9,10]$ :

$$
L^{+}(\mathbf{x})=\mathbf{K}_{1_{i}}(\mathbf{x}) \frac{\partial}{\partial x_{i}}+\mathbf{K}_{2 i j} \frac{\partial^{2}}{\partial x_{i} \partial x_{i}},
$$

where $\mathbf{K}_{1_{i}}$ (x) and $\mathbf{K}_{2 i j}(\mathbf{x})$ were introduced before and $L^{+}(\mathbf{x})$ is a self ad-joint to FPK operator [9].

Now from (7) it is possible to define the differential equation for the average of any function $g(\mathbf{x})$ as:

$\frac{d<g(\mathbf{x})>}{d t}=<L^{+}(\mathbf{x}) g(\mathbf{x})>$

where $<>$ is an averaging operator.

Here we will present this technique (for simplicity) only for one-dimensional case.

Let $g(x)=x, x^{2}, x^{3}, \ldots$ and substituting it into (8), one gets for $\boldsymbol{t} \rightarrow \infty$ :

$$
<K_{1}(\mathrm{x}) g^{\prime}(\mathrm{x})>+<K_{2}(\mathrm{x}) g^{\prime \prime}(\mathrm{x})>
$$

Then it immediately comes:

$$
\begin{aligned}
& <K_{1}(\mathrm{x})>=0 \\
& 2<\mathrm{x} K_{1}(\mathrm{x})>+<K_{2}(\mathrm{x})>=0 \\
& \quad \vdots \quad \vdots \quad \vdots \\
& s<\mathrm{x}^{s-1} \cdot K_{1}(\mathrm{x})>+\frac{s(s-1)}{2}<\mathrm{x}^{s-2} \cdot K_{2}(\mathrm{x})>=0
\end{aligned}
$$

Now, with the help of [9] with application of "cumulant brackets" (see Appendix) one can get:

$$
\begin{aligned}
& <K_{1}(\mathrm{x})>=0 \\
& 2<\mathrm{x}, K_{1}(\mathrm{x})>+<K_{2}(\mathrm{x})>=0 \\
& <\mathrm{x}, \mathrm{x}, K_{1}(\mathrm{x})>+<\mathrm{x}, K_{2}(\mathrm{x})>=0
\end{aligned}
$$

${ }^{2}$ Here we apply the definition for kinetic coefficients in Stratonovich form.
Generalizations for multidimensional case are straight forward [9]:

$$
\begin{gathered}
<\mathbf{K}_{i j}(\mathbf{x})>=0, \\
2\left\{<\boldsymbol{x}_{i}, \mathbf{K}_{1 j}(\mathbf{x})>\right\}_{s}+<\mathbf{K}_{2 i j}>=0, \\
3\left\{<\boldsymbol{x}_{i}, \boldsymbol{x}_{j}, \mathbf{K}_{1 \beta}(\mathbf{x})>\right\}_{s}+3\left\{<\boldsymbol{x}_{1}, \mathbf{K}_{2 j \beta}>\right\}_{s}, \\
\vdots \\
\sum_{l=1}^{n} C_{n}^{l}\left[\left\{<\boldsymbol{x}_{1}, \boldsymbol{x}_{2}, \ldots, \boldsymbol{x}_{n-l}, \mathbf{K}_{1 n-l+1}(\mathbf{x})>\right\}_{s} \ldots\right. \\
\left.\boldsymbol{x}_{1}, \boldsymbol{x}_{2}, \ldots, \boldsymbol{x}_{n-l}, \mathbf{K}_{2 n-l+1}>=0\right],
\end{gathered}
$$$$
\text { where } i \neq j, \beta=\overline{1, n} \text {. }
$$

We can evidently see from (9), that if $\forall \varepsilon_{i j} \rightarrow 0$, then the second summand in (9) tends to zero and the equations in (9) tend to the so-called "degenerated cumulant equations".

Hence, the degenerated cumulant equations have the following form:

$$
\begin{gathered}
<\mathbf{K}_{1 i}(\mathbf{x})>=0, \\
2\left\{<x_{i}, \mathbf{K}_{1 j}(\mathbf{x})>\right\}_{s}=0, \\
3\left\{<x_{i}, x_{j}, \mathbf{K}_{1 \beta}(\mathbf{x})>\right\}_{s}=0,
\end{gathered}
$$$$
\text { where } i, j, \beta=\overline{1, n} \text {. }
$$

Equations (9) and (10) are nonlinear algebraic equations and $\left\langle x_{i}, x_{j}, \ldots, x_{\beta}\right\rangle$ are the so-called "cumulant brackets" abbreviated representation for any cumulant $[9,10]$.

$A\{x, y, \ldots, z\}_{s}$ is an abbreviation of the Stratonovich symmetrization brackets ( $A$ is an integer) and represents the sum of all possible $A$ permutations of the arguments inside the brackets ([9] see Appendix A2).

Essentially equations (9) and (10) represent a set of nonlinear algebraic equations and this set, in general, is not closed, but it is always possible to cut the set of cumulants by neglecting all cumulants with order $s>m$.

The equations (10) have to be sequentially solved first for each component of $\mathbf{x}=\left[x_{1}, x_{2}, \ldots, x_{n}\right]^{\mathrm{T}}$ (first line), next for couples of components $\left\{x_{i}, x_{j}\right\}_{i, j=1}^{n}$ (second line), and then for triplets $\left\{x_{i}, x_{j}, x_{\beta}\right\}_{i, j, \beta=1}^{n}$ (third line), etc.

The way to do it is to "open" the cumulant brackets as shown in ([9] chapter 4 Appendix A2). To illustrate the procedure described above, we apply this material to the analysis of the Rössler attractor.

\section{ROSSLER ATTRACTOR} form:

Equation (1) for the Rössler attractor has the following

$$
\begin{aligned}
& \dot{x}=-y-z, \\
& \dot{y}=x+a y, \\
& \dot{z}=b+z x-z c,
\end{aligned}
$$

where $a, b, c$ are the parameters of the attractor, $\mathbf{x}=[x, y, z]^{\mathrm{T}}$.

Thus, the first kinetic coefficients for (11) are: 
$\mathbf{K}_{1,1}(x)=-y-z$,

$\mathbf{K}_{1,2}(x)=x+a y$,

$\mathbf{K}_{1,3}(x)=b+z x-z c$.

Introducing (11) and (12) into (10) for the first component " $x$ ", one gets:

$$
\left.\begin{array}{r}
\chi_{1}^{y}=<y>=-<z>=-\chi_{1}^{z} \\
\chi_{1,1}^{x, z}=-\chi_{1,1}^{x, y} \\
\chi_{2, y}^{x, y}=-\chi_{2,1}^{x, z} \\
\chi_{3,1}^{x, y}=-\chi_{3,1}^{x, z} \\
\chi_{4,1}^{x, y}=-\chi_{4,1}^{x, z}
\end{array}\right\}
$$

Then, for the second component " $y$ ":

$$
\left.\begin{array}{rl}
\chi_{1}^{x}=<x>=- & <y>=-a \chi_{1}^{y} \\
\chi_{2}^{y} & =-\frac{1}{a} \chi_{1,1}^{x, y} \\
\chi_{3}^{y} & =-\frac{1}{a} \chi_{2,1}^{y, x} \\
\chi_{4}^{y} & =-\frac{1}{a} \chi_{3,1}^{y, x}
\end{array}\right\}
$$

Finally for the third component " $z$ ":

$$
\left.\begin{array}{r}
\chi_{1,1}^{x, z}=\chi_{1,1}^{z, x}=c<z>=-<z><x> \\
\chi_{2}^{y}=\frac{c<z>-a<z>^{2}}{a}
\end{array}\right\}
$$

On the other hand:

$\chi_{2}^{\mathrm{y}}=\frac{\chi_{2}^{\mathrm{x}}}{<\mathrm{z}>}$.

Then, going on with two components $\{x, y\}$, one can get:

$\chi_{3}^{y}=\frac{2 \chi_{1,2}^{y, x}}{1+a} \neq 0$

i.e. PDF of $y(t)$ is asymmetrical.

With two components $\{x, z\}$ it follows: $\chi_{3}^{z} \neq 0$, i.e. PDF of $z(t)$ is asymmetrical as well.

The cumulants: $\chi_{1,1}^{z, y}, \chi_{1,1}^{z, x}$, etc., are very important because they show the degree of "similarity" for the chaotic signals, $x(t), y(t)$ and $z(t)$

Particularly they are:

$$
\begin{gathered}
\chi_{1,1}^{z, y}=a<y>\chi_{2}^{y}-\frac{<y>}{a} \approx a<y>\chi_{2}^{y} ; \\
\chi_{1,1}^{z, x} \cong<z>c,
\end{gathered}
$$

when $\mathrm{c}>>\mathrm{a}$.

Together with the previously defined $\chi_{1,1}^{x, y}$ those two cumulants illustrate the measure of linear statistical dependence between the components of the attractor.

Strictly speaking, as it follows from (13), (14), all components of the Rössler attractor have an asymmetry in the PDF's, but the degree of its asymmetry is different: for " $z$ " component: $\chi_{3}^{z} \approx \frac{\chi_{2}^{z}}{c}$, while $\chi_{3}^{x} \approx 0$ (see also $\chi_{3}^{x} \approx 0$ above), i.e. $\gamma_{3}^{z} \neq \gamma_{3}^{y} \neq 0$.

So, $x(t)$ has practically a symmetrical PDF, but the PDF's for $y(t)$ and $z(t)$ are significantly asymmetric.

At the same time, the mean value $\langle x\rangle$ is less than $\langle y\rangle$ and it is possible for the qualitative calculus to assume, that $\langle x\rangle \approx 0$. For the case of $\langle y\rangle$, the last assumption is not valid; the same for $\frac{\langle x\rangle}{a}$, etc.

Next, it follows, that $\chi_{4}^{x} \approx\left(\chi_{2}^{x}\right)^{2} ; \chi_{4}^{y} \approx\left(\chi_{2}^{y}\right)^{2}$; and $\gamma_{4}>0$. Then $W(x)$ and $W(y)$ are "sharper" than the Gaussian PDF $W_{G}(x)$ with the same first two cumulants. Note, that the last one shows the importance of the calculus of the variance of the chaos as well.

So, careful analysis even of the first four cumulants gives an interesting qualitative and quantitative knowledge regarding the statistical characterization of chaos generated from the Rössler attractor. To the best of our knowledge this has not been reported previously,

In the same way all necessary cumulants of the higher order can be found, from (8) (all notations for cumulants applied in this section are presented in the Appendix).

\section{NUMERICAL RESULTS AND DISCUSSIONS}

Let us consider the results of simulations of the Rössler attractor with the following parameters: $a=0.2, b=0.2, c=$ $5.7[14,17]$.

Note that as it was commented above, each component of the attractor achieves its stationary conditions with different time constant. At this step of the research it was empirically found (from simulations), that the " $x$ " components reach their stationary state faster than " $y$ " and " $z$ " components (it also follows from the inverse eigenvalues shown in the following section). Therefore for comparison with the analytical results it is reasonable to apply data from the simulations, when the PDF's clearly achieve their stablestate shapes.

Keeping this in mind, let us make brief comparisons between analytical and simulation results. Based on the analytical results, which were presented in section II, we will consider mainly the comparisons of the first cumulants, as they are very important for practice.

From simulations it was found:

$<x>=0.17,\langle y>=-0.76,<z>=0.75$.

One can see from (13) and (14) the almost exact coincidence with theory.

Then, from (15) it follows, $\chi_{2}^{\mathrm{y}}=17.8$ and simulation gives $\chi_{2}^{y}=18.9$ (error is about $5.8 \%$ ). 
From (14) $\langle x>=0.13$ and $K x>|<K y>|:\langle y\rangle=0.17$ from (16) it comes: when $\chi_{2}^{y}=13.3, \chi_{2}^{y}=17.8$ (simulation gives almost the same).

Both PDF's for components " $y$ " and " $z$ " are not symmetrical. From (14) it follows, that PDF's for " $x$ " and " $y$ " are in some sense oppositely asymmetric, in contrary to Lorenz attractor (see [7]).

It can be seen, that besides of a good accuracy of the analytical prediction for cumulants, for complete calculus of the cumulants it is mandatory to provide the calculus of the variances: $\chi_{2}^{x}, \chi_{2}^{y}, \chi_{2}^{z}$, as high order cumulants depend on them and the above presented approach does not allow calculating them right away.

In Figs. (1-3) the histograms for PDF's of the " $x "$, " $y$ " and " $z$ " components of the Rössler attractor are represented for normalized values.

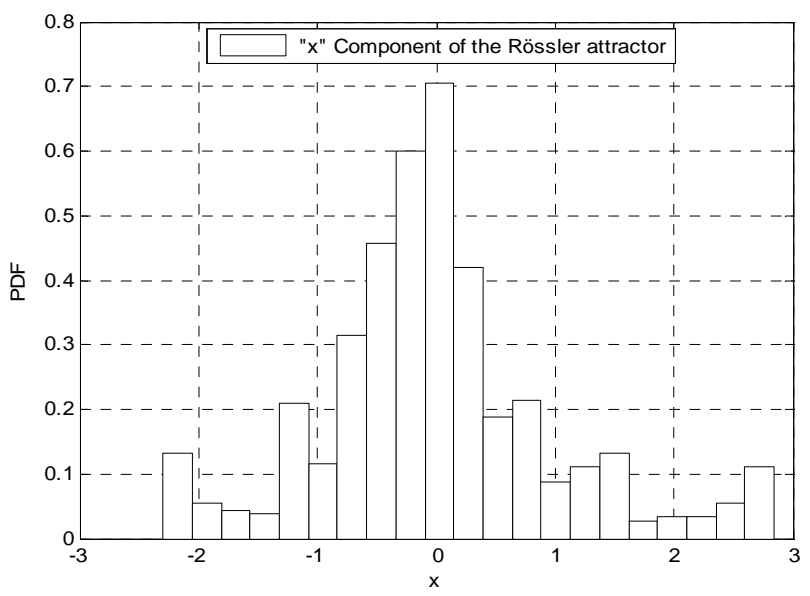

Fig. (1). " $x$ " component of the Rössler attractor.

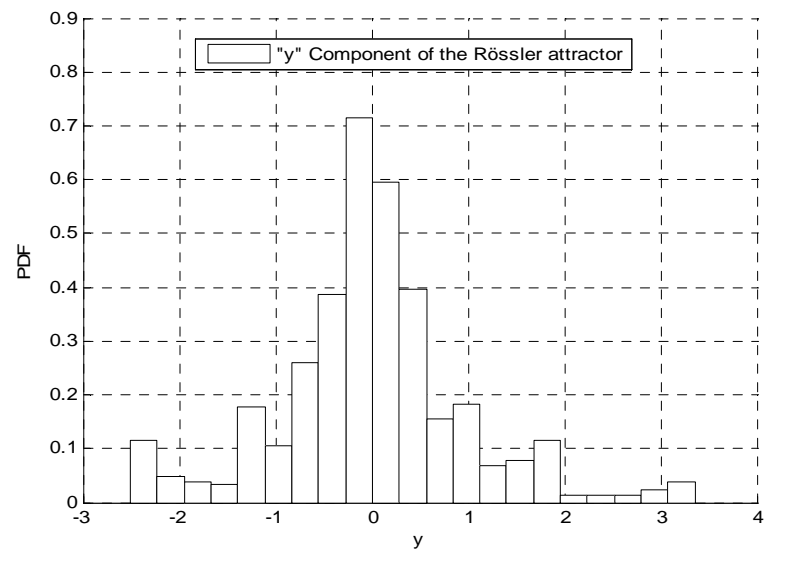

Fig. (2). " $y$ " component of the Rössler attractor.

One can see, that the components " $x$ " and " $y$ " (contrary to Lorenz attractor [8]) are "oppositely" asymmetric, and have unimodal PDF's with $\gamma_{4}>0$, i.e. the vertices of the distributions are "sharper", than the Gaussian ones. Next, one can see that the component " $\mathrm{z}$ " can be approximated by means of a delta- function.
For " $\mathrm{x}$ " and " $y$ " components we approximate the PDF histograms by means of the Laplace distribution defined by:

$f(x)=\frac{1}{2 \lambda} \exp \left\{-\frac{|x-\mu|}{\lambda}\right\}$.

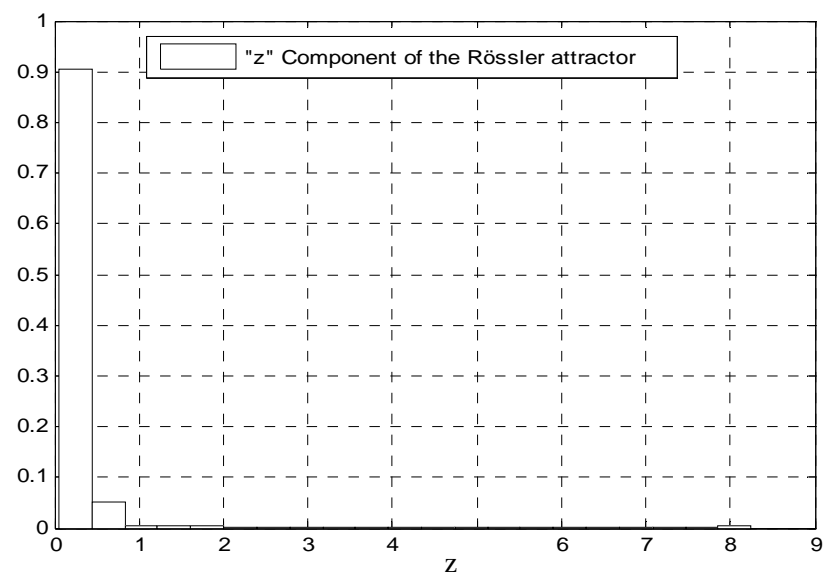

Fig. (3). " $z$ " component of the Rössler attractor.

This distribution is characterized by location $\mu$ and scale $\lambda$ parameters. The use of Laplace distribution allows to make a right description for the " $x$ " and " $y$ " components of the Rössler attractor as it is observed in the Figs. $(\mathbf{4}, \mathbf{5})$.

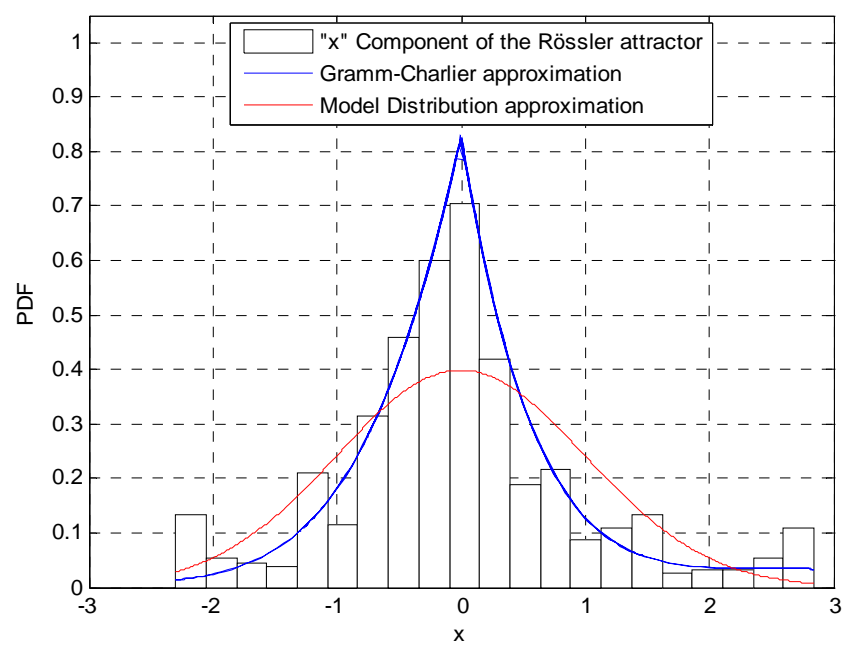

Fig. (4). " $x$ " component of the Rössler attractor.

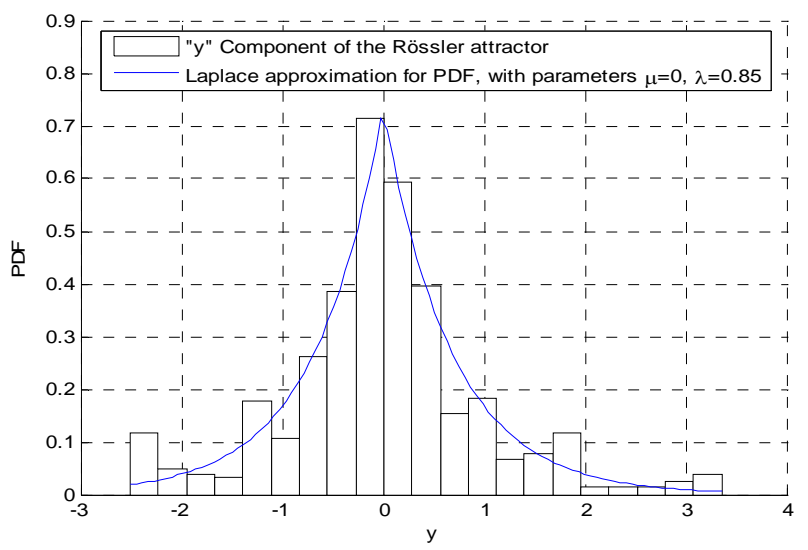


Fig. (5). " $y$ " component of the Rössler attractor.

Then, as it follows from Figs. $(4,5)$, the (PDF), for the " $x$ " component of the Rössler attractor is approximated by a Laplace distribution with the local parameter $\mu=0$ and scale parameter $\lambda=1.1$ and for the " $y$ " component with local parameter $\mu=0$ and scale parameter $\lambda=0.85$.

In Figs. $(6,7)$, we apply the Kolmogorov- Smirnoff goodness of fit test with a significance level $\alpha=0.05$, in order to examine whether the accuracy of the PDF of the " $x$ " and " $y$ " components of the Rössler attractor and its approximation using the Laplace distribution are adequate or not. As it can be seen from the Figs. $(6,7)$, the approximation can be considered as acceptable. Utilization of the Chi-Square test gives the same results, which are not presented here.

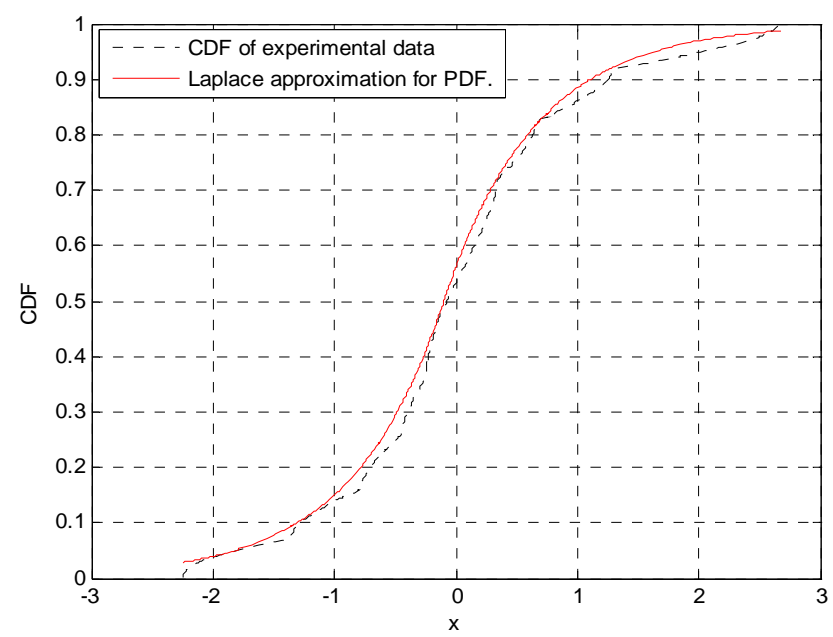

Fig. (6). CDF of the " $x$ " component using the Laplace approximation.

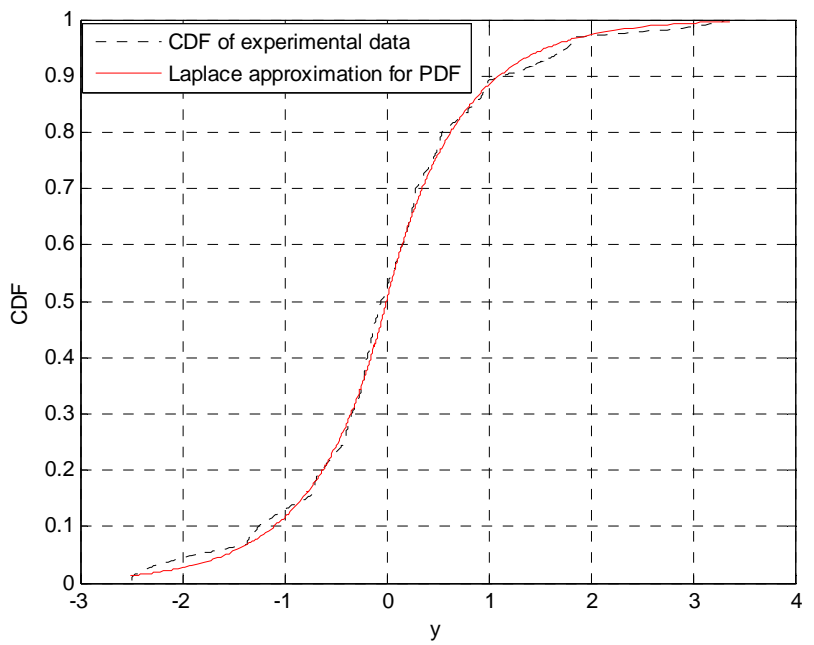

Fig. (7). CDF of the " $y$ " component using the Laplace approximation.

In Figs. $(8,9)$, the results of the approximation of the PDF for the " $x$ " and " $y$ " components of the Rössler attractor by means of the Gramm-Charlier series and the model distribution (5) (both with $\gamma_{4}>0$ ) are represented.

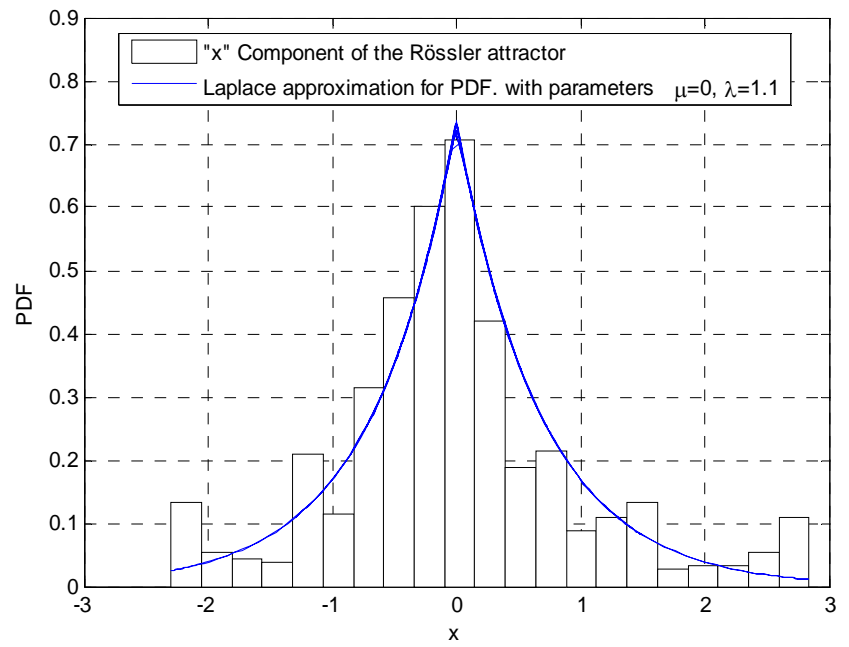

Fig. (8). The " $x$ " component of the Rössler attractor with the Laplace and model distribution approximation.

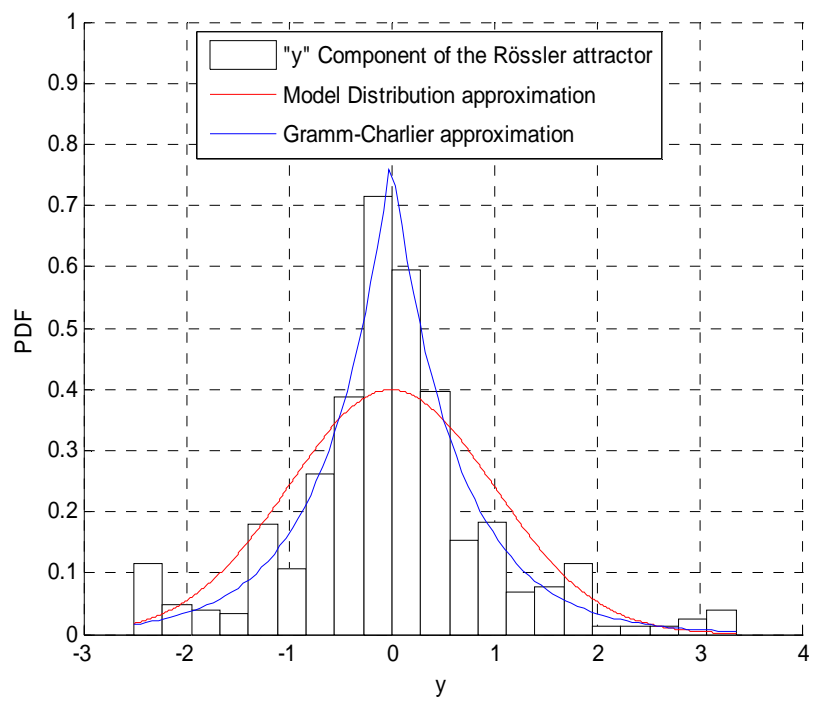

Fig. (9). The " $y$ " component of the Rössler attractor with the Laplace and model distribution approximation.

One can see, as it was commented before, that for this case the Gramm-Charlier approximation is more precise, than the expression (5), because of $\gamma_{4}>0$.

\section{APPROXIMATE APPROACH FOR VARIANCE CALCULATION}

By considering the results presented in previous sections we realize that the approximations obtained by applying the cumulant method are rather good.

As it was mentioned above, the first cumulants for the Rössler attractor depend on the evaluation of the variance $\chi_{2}$ and this evaluation can not be performed directly from the degenerated cumulant equations approach.

Thus, in the following we present an approximate approach for the variance evaluation, based on the concept of the Kolmogorov-Sinai $H_{k-s}$ entropy $[14]^{3}$, with the conjecture,

${ }^{3}$ Considering that K-S entropy can not be exactly calculated, it is impossible by using this method to obtain an exact result for variance. Nevertheless, 
that variance as a measure of the "fluctuations" of the process has some influence on the information measure as well.

The $H_{k-s}$ entropy is defined by the sum of the Lyapunov exponents for the non-linear system of the attractor ([14], page 122). For a linear matrix $\mathbf{A}$ the Lyapunov exponents are defined by its eigen-values ([17], pages 542-543).

In the proposed methodology first we compare the differential entropy obtained from the PDF approximation for a component of the Rössler attractor, with the $H_{k-s}$ entropy computed through the parameters of the attractor (Lyapunov exponents).

Note, (see in the following) that in the framework of this analysis $H_{k-s}$ coincides with the Kolmogorov deferential entropy of the PDF ([17], pages 542-543, 839).

The proposed methodology can be summarized as follows.

1. The non-linear system describing the chaotic behavior of the Rössler attractor has to be statistically linearized ([17], page 760).

2. The eigen-values must be found from the coefficients matrix formed by the linearized system.

3. Once the eigen-values have been obtained, the $H_{k-s}$ for the dynamic system can be estimated as follows [17]:

$\log \left|\lambda_{\max }\right|<H_{k-s} \leq \sum_{j=1}^{m} \log \left|\lambda_{j}\right|$,

where, $j=\overline{1, m}, \lambda_{j}$-is the $j$-th eingen-value of the linear matrix and $\log \left|\lambda_{j}\right|$-is the $j$-th Lyapunov exponent for the linear matrix ([17], page 542).

4. On the other hand the Kolmogorov deferential entropy is:

$$
h_{d i f}=-\int_{\infty}^{-\infty} W(x) \log [W(x)] d x
$$

where $W(x)$ is the PDF of the output signals whose parameters have to be represented through $\chi_{2}$.

5. Then, we create an algebraic equation depending on the variance according to steps 3 and 4 .

6. Solving equation from steps 3 and 4 , we can have now a solution for variance.

It is worth mentioning, that as long as the whole Kolmogorov-Sinai entropy is addressed to the given component of the Rössler attractor, then the value of the variance obtained from the approach is actually its upper bound. The lower bound can be found applying the left hand side of the inequality (18).

Here we present the results of the application of this method for the Rössler attractor considering only the $x$ component as an example.

From equation (11) we can obtain a linearized system as it was mentioned in step 1. From step 2, we obtain the coefficient matrix $\mathbf{A}$ of this linearized system as:

assuming that for practical purposes an error about $10-20 \%$ is acceptable, we are able to apply this method.
$\mathbf{A}=\left|\begin{array}{ccc}0 & -1 & -1 \\ 1 & a & 0 \\ 0 & 0 & -c\end{array}\right|$

Matrix A has a determinant different from zero, for this reason we can obtain eigen-values, considering $a=0.2$ and $c=5.7$. The characteristic equation becomes:

$\lambda^{3}-(a-c) \lambda^{2}-(a c-1) \lambda+c=0$,

where $\lambda_{1}=-5.7, \lambda_{2}=0.1+j$ and $\lambda_{3}=0.1-j$.

Note that the main eigen-value $\lambda_{1}$ is equal to the parameter $c$ and its inverse value is the lowest time constant (see comments above).

Having the results for the eigen-values, and substituting $\lambda_{l}$ into (16), we can obtain a numeric value for entropy as $H_{k \text { - }}$ $\approx 1.74$.

And for steps 3 and 6 it follows:

$\chi_{2}^{x}=\frac{\exp (3.48)}{2 e^{2}}=2.1$.

From Fig. (4) it follows, that $\chi_{2}^{x} \approx 2$, and the error is $5 \%$.

Next, let us make some generalizations. In the previous material it was assumed that the parameters of the Rössler strange attractor are predefined as well as the PDFs for the output signals.

Here we will consider a more general case: let us suppose that the set of parameters of the strange attractor and the PDFs are not predefined, but the chaotic regime of the attractor (spiral or punnel chaos) is established.

Then one can apply for the PDF choice the so-called "Maximum Entropy Method (MEM)" [15].

If the attractor of interest can be characterized by the symmetric PDFs, then the Gaussian PDF can be applied as a MEM distribution (for " $x$ " component).

From the MEM principle it follows, that for a given $H_{k-s}$ for Gaussian PDF, the evaluated $\chi_{2}$ has to be a lower boundary for its true value.

It follows from (18), that the variance $\chi_{2}$ obeys the following inequality:

$\chi_{2} \geq \frac{10^{2 \mathrm{H}_{\mathrm{k}-\mathrm{s}}}}{2 \pi \mathrm{e}}$.

For the Rössler attractor $\chi_{2}^{x} \geq 1.8$ (the true value is 2 and the error is $10 \%$ ).

Next, let us make some concluding comments regarding the material of this section.

It was shown that through the ordinary equations of strange attractors and their statistical linearization it is possible to obtain analytical estimations of the Lyapunov exponents of the non-linear dissipative system describing the attractor. After the Lyapunov exponents are found it is possible to get a well known approximation for the Kolmogorov-Sinai entropy $H_{k-s}$. 
On the other side, applying the attractors PDFs for the components of interest with its analytical approximation, it is possible to find the Kolmogorov defferential entropy $\left(h_{\text {dif }}\right)$ which practically coincides with $H_{k-s}$ for the strange attractors under consideration. Finally, by solving a simple quadratic algebraic equation one can find $\chi_{2} \geq 0$.

The approach shows acceptable accuracy for engineering evaluation of $\chi_{2}$ (less than 20\%) and it is completely analytical. Why it happened?

It seems, that all depends on the stochastic set of the attractor, the dimension of the phase space of the attractor, etc. (for details see [14, 17], etc.).

For example if the dimension of the stochastic set $\mathrm{D}$ for the strange attractor is more that two, almost all phase trajectories, that constitute the strange attractor are localized in a very thin layer, i.e., it can be approximately represented by a one dimensional Poincare mapping (details can be found in $[14,18])$. It is actually true for Chua, Lorenz, Rössler etc. strange attractors as well (see [2, 18], etc).

So, any non-linear dissipative system (strange attractor) with a dimension $\mathrm{D}$ equal or more than two represents a onedimensional Poincare mapping (one dimensional dynamics) and one of the consequences of this is the similarity between $H_{k-s}$ and the Kolmogorov differential entropy. Therefore, the acceptable accuracy of the proposed approach might be, in some sense, predicted.

We would like to emphasize, that the above presented methodology can be applied to arbitrary attractors with some caution: first, the variance has to exist (see $z$-component of the Rössler attractor, as an exception, at least in the framework of our approximations); next, the attractor has to be "strange", etc.

Therefore, the exact variance evaluation approach has to take into account the details of the nonlinear dynamics of the system (see [15], for example) in a much more explicit way, than it was done here.

Finally, as it follows from the above comments, the problem of establishing the dependence of $\chi_{2}$ on the parameters of the strange attractors is not trivial at all, and to the best of our knowledge no analytical results have been reported yet in this regard (only numerical integration for (9)).

\section{MODELING OF RADIO FREQUENCY INTERFERENCE OF LAPTOPS AND DESKTOPS WITH OUTPUT SIGNALS OF THE RÖSSLER ATTRACTOR}

Now, we present the results of the application of the statistical analysis of the Rössler attractor to the modeling of RFI caused by the PCIe serial bus of high speed interconnects of laptops and desktops.

Let us consider a PCIe bus, which is widely used in computers to communicate at high speed data rates. Specifically, the broadband emissions generated by high speed digital interconnects input-output (I/O) bus transactions associated with graphics benchmark (SW,
3Dmark) are a major contributor of the non-Gaussian radio interferences to the PCIe platforms. Those interferences were experimentally investigated through the Intel measurement set up system.

The measurement system consists of a high speed data capture system: RF gain and analog to digital converter (ADC). In addition, a low loss RF cable is used to connect a RF horn antenna.

The methodology for the measurement can be summarized as follows.

1. The equipment used for measurements is put inside a Faraday cage, and then the laptop is wirelessly connected to another computer, which is located inside a compartment that allows isolation of the radio signals; the door compartment can be opened or closed at any time and so, the communication is intermittent.

2. The RFI measure originates from a laptop, Device Under Test (DUT), with all its system assets (hardware/ PCIe buses, LCD/Graphics and wireless systems) operating under normal traffic conditions.

3. The radiated emission level measurement is $-100 \mathrm{dBm}$ over a $20 \mathrm{MHz}$ bandwidth, with an (ADC) 40 Msps. This is the minimum required level of the $\mathrm{WiFi}$ radio subsystem (i.e. $802.11 \mathrm{a}$ and $802.11 \mathrm{~g}$ IEEE Standards). Therefore, the modeled statistics are representative of the conditions to be experienced by the platform's wireless subsystems.

4. The measurement conditions are:

ON/OFF: The screen graphics of the laptop, where the measurement was carried out, are activated and deactivated.

CLOSED/OPEN: The compartment door (where the computer with a wireless card is) is opened or closed.

Following the above mentioned procedure for measurements the experimental data of the interference of laptops and desktops at $20 \mathrm{MHz}$ were analyzed using histograms, PDFs and output signals of the " $x$ " and " $y$ " components of the Rössler attractor.

As before, the data are normalized as a random variable $\dot{x}=(x-\mu) / \sigma$ where $\mu$ and $\sigma^{2}$ are the mean and variance respectively.

Considering the application of the " $x$ " component of the Rössler attractor as a RFI model with its PDF shown in Fig. (10), one can see that the analytical and experimental results are practically identical. The analytical approximation corresponds to the Laplace distribution function and the correspondent cumulants coincide as well.

Let us take another example of RFI from PCIe. For this example, measurements were taken from PCIe buses at 20 $\mathrm{MHz}$ (again). In this case the " $y$ " component of the Rössler attractor was used as a RFI model with its PDF shown in the Fig. (11). One may see that analytical and experimental results are very similar. 
It is necessary to examine whether the accuracy of the RFI modeled through the PDFs of the " $x$ " and " $y$ " components of the Rössler attractor and the analytical approximation of each of them by the Laplace distribution is adequate or not.

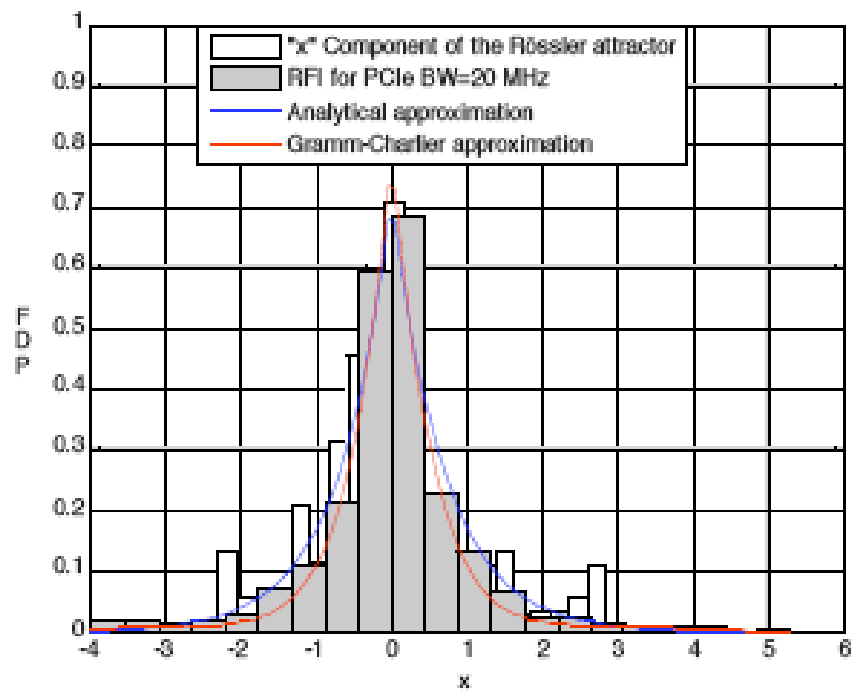

Fig. (10). " $x$ " component of the Rössler attractor and RFI for PCIe with analytical approximation.

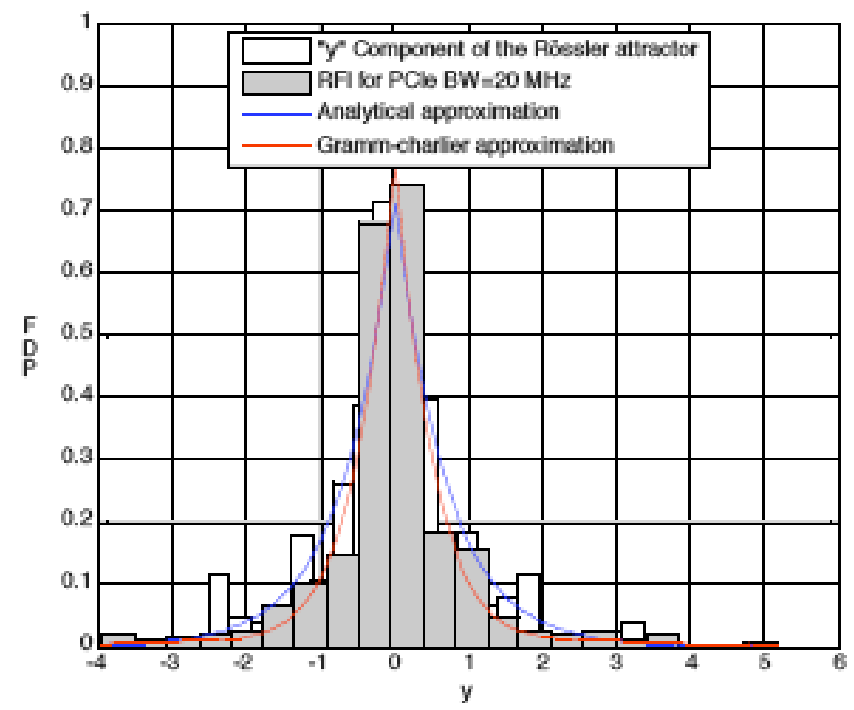

Fig. (11). " $y$ " component of the Rössler attractor and RFI for PCIe with analytical approximation.

In the same way as in section III this was achieved by applying the Kolmogorov-Smirnoff and Chi-Square goodness of fit test, both tests validate our approximation, even though the Chi-Square test indicates a better accuracy.

From this material it follows that the output signals of the Rössler attractor can be successfully applied for the statistical modeling of the RFI in high speed interconnects. More detailed study about this will be presented in another publication.

\section{CONCLUSIONS}

The material presented above allows us to make the following conclusions:
1. The degenerated cumulant equations approach applied to statistical analysis of the Rössler attractor provide rather accurate results for the predicted cumulants;

2. Almost exact formulas for the first four cumulants, which can be applied both for orthogonal and model distribution representations of the PDF for the chaotic signals were obtained;

3. The PDF's for the " $x$ " and " $y$ " components of the Rössler attractor were successfully approximated by the Laplace PDF and by orthogonal series representations, which in turn use results of the cumulant analysis;

4. An original approach for the variance calculation, based on the Kolmogorov-Sinai entropy method together with the Lyapunov exponents evaluation was presented;

5. Theoretical results of the stochastic analysis of the Rössler attractor were applied for the practical modeling of the RFI from laptops and desktops.

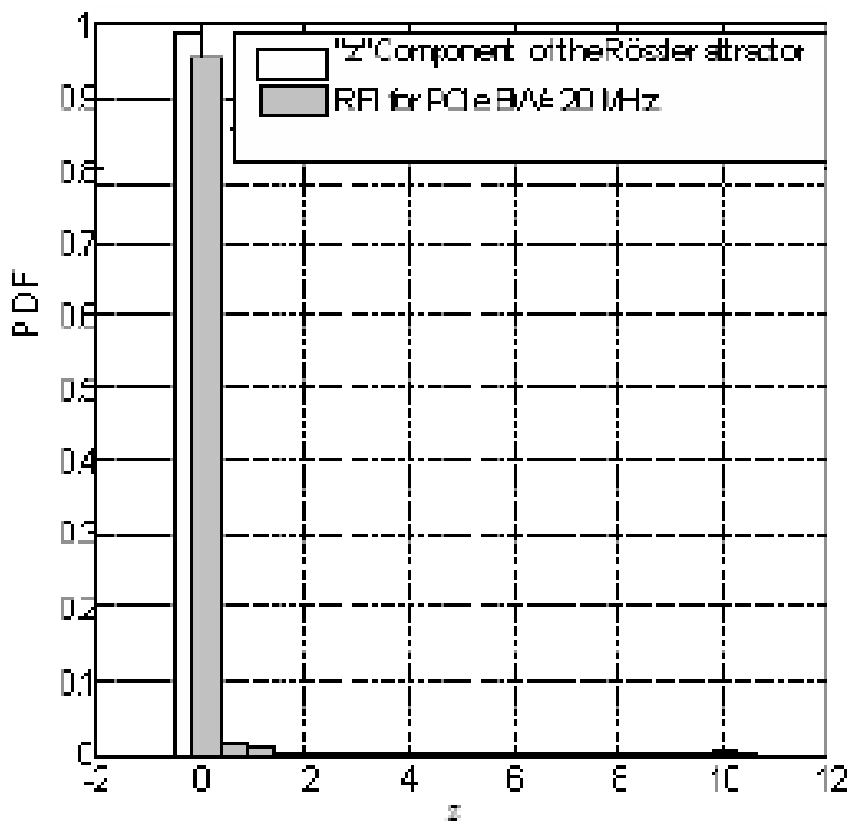

Fig. (12). "z" component of the Rössler attractor and RFI for PCIe.

\section{ACKNOWLEDGEMENTS}

The authors are pleased to pass their gratitude to Fernando Ramos and Oscar Filio for their help in critical revision of this paper.

\section{APPENDIX}

The concept of cumulant brackets was introduced as an abbreviated representation for any cumulant, i.e.

$$
\begin{aligned}
& \chi_{m_{1}, m_{2}, \ldots, m_{n}}^{\xi_{1}, \xi_{2}, \ldots, \xi_{n}}=<\underbrace{\xi_{1}, \ldots, \xi_{1}}_{m_{1}}, \underbrace{\xi_{2}, \ldots, \xi_{2}}_{m_{2}}, \cdots, \\
& \underbrace{\xi_{n}, \ldots, \xi_{n}}_{m_{n}}>\equiv<\xi_{1}^{\left.\xi_{1}\right]}, \xi_{2}^{\left[m_{2}\right]}, \ldots, \xi_{n}^{\left.\xi_{n}\right]}>,
\end{aligned}
$$


where $\xi_{1}$ appears inside the brackets $m_{1}$ times, $\xi_{2}$ appears $m_{2}$ times, and so on; for example, the third cumulant is

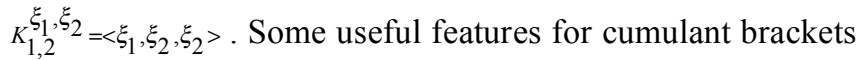
can be found in [9].

By means of cumulants brackets it is possible to formalize the operations between random variables and their transformations: linear and nonlinear, inertial and noninertial (see [10], also [9], for some generalizations).

For the above presented material some features for cumulant brackets need to be taken into account $[9,10]$ :

1. $<\xi, \eta, \ldots, \omega>$ is a symmetric function of its arguments.

2. $<a \xi, b \eta, \ldots, g \omega>=a \cdot b \cdot \cdots, g<\xi, \eta, \ldots, \omega>$, where $a, b, \ldots, g$ are constants.

3. $<\xi, \eta, \ldots, \theta_{1}+\theta_{2}, \ldots, \omega>=<\xi, \quad \eta, \ldots, \theta_{1}, \ldots, \omega>+$ $<\xi, \eta, \ldots, \theta_{2, \ldots, \omega>}$.

4. $\langle\xi, \eta, \ldots, \theta, \ldots, \omega>=0$, if $\theta$ is independent of \{ $\xi, \eta, \ldots, \omega\}$.

5. $<\xi, \eta, \ldots, a, \ldots, \omega>=0$.

6. $<\xi+a, \eta+b, \ldots, \omega+g>=<\xi, \eta, \ldots, \omega>$.

In addition to cumulant brackets it is necessary to introduce here the concept of Stratonovich symmetrization brackets: symmetrization brackets together with the integer number in front of the brackets represent the sum of all possible permutations of the arguments inside the brackets. For example, the operator $3\left\{<\xi_{1}>\cdot<\xi_{2}, \xi_{3}>\right\}_{\mathrm{s}}$ means that:

$3\left\{<\xi_{1}>\cdot<\xi_{2}, \xi_{3}>\right\}_{s}=<\xi_{1}>\cdot<\xi_{2}, \xi_{3}>\cdots$

$+<\xi_{2}>\cdot<\xi_{2}, \xi_{3}>+<\xi_{3}>\cdot<\xi_{1}, \xi_{2}>$,

where \{\}$_{s}$ is the notation for the Stratonovich symmetrization brackets.

Rules for manipulations with cumulant brackets can be found in A2 [9].

Actually, the relations between moments and cumulants for the same distribution $W(x)$ are well known (see, for instance, references $[9,10]$, etc.); the following notation is also well known: $\langle g(x)\rangle,\left\langle\xi_{1} \cdot \xi_{2}\right\rangle$, which denotes the operator of statistical average for $g(x)$ and for the product of two random variables $\xi_{1}$ and $\xi_{2}$, respectively. Following [9, 10] we call this operator $<\cdot>$ as moment brackets, so the formal difference between moment and cumulant brackets is, that the first one contains a 'dot' between random variables (usually it is skipped), and the second one contains a 'comma' between variables.

\section{ACKNOWLEDGEMENT}

This work was supported by Intel Corporation through the grant "Intel-VK".

\section{REFERENCES}

[1] M. Hasler, G. Mazzini, and M. Ogorzalek, "Applications of nonlinear dynamics to electronics and information engineering", Special Issue, Proceedings of the IEEE, vol. 90, no. 5, pp.631-639, May 2002.

[2] R. Badii and A. Politi, "Statistical Description of Chaotic Attractors: The Dimension function", J. Statist. Phys., vol. 40, no. (5/6), May 1985.

[3] A. Lasota, and M. Mackey, Chaos Fractal and Noise. Berlin, Springer: 2003.

[4] W. Horton, and Y. H. Ichikawa, "Chaos and Structures in Nonlinear Plasmas", Singapure, World Scientific 1996.

[5] X. Abel and W. Schwartz, "Chaos communications principles, schemes, and system analysis", Proceedings of the IEEE (Special Issue on Applications of Non-linear Dynamics to Electronics and Information Engineering), vol. 90, no. 5, 691-710, May 2002.

[6] V.S. Anischenko, T.E Vadivasova, G.A Okrokvertskhov, and G.I Strelkova, "Mixing and spectral-correlation properties of chaotic and stochastic systems: numerical and physical experiments", New J. Phys., vol.7, no.76, pp. 1-29, May 2005.

[7] V.S. Anischenko, T.E Vadivasova, G.A Okrokvertskhov, and G.I. Strelkova "Statistical properties of dynamical chaos", PhysicsUspehi, vol.48, no.2, pp. 151-166, February 2005.

[8] V. Kontorovich, "Applied Statistical analysis for strange attractors and related problems", Math. Methods Appl. Sci., vol. 30, pp. 1705-1717, April 2007.

[9] S. Primak, V. Kontorovich and V. Lyandres, Stochastic Methods and their Applications to Communications. Stochastic Differential Equations Approach: John Wiley \& Sons, Ltd. 2004.

[10] A. Malakhov, Cumulant analysis of Random Non-Gaussian Process and their Transformation, Sovetskoe Radio: Moscow, 1975 (in Russian).

[11] M. Mijangos, V. Kontorovich and J. Aguilar-Torrentera, "Some statistical properties of strange attractors: engineering view", J. Phys: Conference Series: 012147, vol. 96, March 2008.

[12] J.P. Eckman, and D. Ruelle, "Ergodic theory and Strange Attractors", Rev. Mod. Phys., vol. 57, no. 3, part 1, pp. 617-656, July 1985.

[13] M. Mijangos, and V. Kontorovich, "Non-Gaussian perturbation modeling through Chua's attractor", XVI International Conference on Electromagnetic Disturbances, (EMD 2006). 27-29 September 2006.

[14] A. Scott, Ed. Encyclopedia of non-linear sciences. N.Y Rontledge, 2005.

[15] A. Papoulis. Probability, Random Variables, and Stochastic Processes. $3^{\text {rd }}$ ed, Boston, M. A, McGraw-Hill, 1991.

[16] V. Kontorovich, S. Primak, and Kh. Almustafa, "On limits of applicability of cumulant method to analysis of dynamic systems with random structure and chaotic behavior", in Proceeding of the III International DSDIS Conference on Engineering Applications and Computer Algorithms, Guelph Canada, May 2003.

[17] B.G. Kukharenco, "Detecting orbits of high periods from numerical solutions of the Rössler system”, Int. IEEE Sym. Phys. Com., pp. 866-871, 2005.

[18] A. Nayfeh and B. Balanchandra. "Applied non-linear dynamics". John Wiley \& Sons, Ltd. 1995.

[19] R. Radev, "Full State Observer for Rössler's System", Int. IEEE Symp. Intelligent Systems, 2002.

[20] M. Rabinovich and D. Trubetskov, "Introduction to Oscillation Theory", "Nauka", Moscow, 1984. (In Russian)

[21] M. Delgado-Restituto, and A. Rodríguez-Vásquez, "Integrated chaos generators", Proceedings of the IEEE (Special Issue on Applications of Non-linear Dynamics to Electronics and Information Engineering), vol.90. no. 5, 747-767, May 2002.

This is an open access article licensed under the terms of the Creative Commons Attribution Non-Commercial License (http://creativecommons.org/licenses/by$\mathrm{nc} / 3.0 /$ ) which permits unrestricted, non-commercial use, distribution and reproduction in any medium, provided the work is properly cited. 